\title{
Predicting Image Aesthetics using Objects in the Scene
}

\author{
Hiya Roy \\ The University of Tokyo \\ Japan \\ hiya@hal.t.u-tokyo.ac.jp
}

\author{
Toshihiko Yamasaki \\ The University of Tokyo \\ Japan \\ yamasaki@hal.t.u-tokyo.ac.jp
}

\author{
Tatsuaki Hashimoto \\ The University of Tokyo \\ Japan \\ hashimoto.tatsuaki@jaxa.jp
}

\begin{abstract}
Analyzing aesthetic quality of images is a highly challenging task because of its subjectiveness. With the exponential rise of digital images in social media, it is of great demand to assess the aesthetics of images for several multimedia applications such as increasing social popularity etc. Previous approaches to address this problem have used hand-designed features or automated features extracted by deep convolutional neural network architectures. In this paper we predict the aesthetics of images by using the inferential information depending on the visual content found in an image. To the best of our knowledge, this is the first attempt to address such problem by using tags predicted. Experimental results show that our proposed method outperforms the traditional machine learning methods and demonstrate competitive performance compared to the state-of-the-art methods of image aesthetics prediction.
\end{abstract}

\section{KEYWORDS}

Image Aesthetics, Convolutional Neural Network, Image tags

\section{ACM Reference Format:}

Hiya Roy, Toshihiko Yamasaki, and Tatsuaki Hashimoto. 2018. Predicting Image Aesthetics using Objects in the Scene. In MMArt\&ACM'18: 2018 International foint Workshop on Multimedia Artworks Analysis and Attractiveness Computing in Multimedia, Fune 11, 2018, Yokohama, Japan. ACM, New York, NY, USA, 6 pages. https://doi.org/10.1145/3209693.3209698

\section{INTRODUCTION}

In recent years, image aesthetics analysis has drawn a significant attention of the computer vision community because of its potential applications in the visual experience domain, such as image enhancement, image cropping, image retrieval, and photo management [5, 9, 13, 18, 23, 25]. Evaluating aesthetics of images using a computational algorithm is a very difficult task for computers because different people perceive beauty in different ways and rate aesthetics of images differently as seen in Figure 1 . Therefore, to deal with the subjectivity of the human's aesthetic evaluation, several machine learning approaches have been proposed over the years $[1,2,5-7,9,11-13,17-19,22,23,25,26,30]$. Most of the early research works have focused on designing intuition based features ranging from low-level features such as color, hue, saturation, etc. [5] to high-level describable image attributes such as compositional, content based or illumination based attributes

Permission to make digital or hard copies of part or all of this work for personal or classroom use is granted without fee provided that copies are not made or distributed for profit or commercial advantage and that copies bear this notice and the full citation on the first page. Copyrights for third-party components of this work must be honored. For all other uses, contact the owner/author(s).

MMArt\&ACM'18, Fune 11, 2018, Yokohama, Japan

(c) 2018 Copyright held by the owner/author(s).

ACM ISBN 978-1-4503-5798-2/18/06.

https://doi.org/10.1145/3209693.3209698
[9] for image aesthetics prediction. However, it is very difficult to choose appropriate features to map the human perception of images to their aesthetic score. To tackle this problem researchers have also adopted generic features such as SIFT and Fisher Vector [19] for predicting aesthetics of images. However, over the past few years, deep convolutional neural network (CNN) based models have shown outstanding performance on various challenging visual recognition tasks $[3,10,15,27,29,31]$ and they have the capability of learning features automatically from image examples in a hierarchical way. Therefore, in the most recent studies researchers have exploited the automated feature learning power of deep convolutional neural network $(\mathrm{CNN})$ for the image aesthetics prediction task, in order to avoid the requirement of domainrelated knowledge to choose appropriate features.

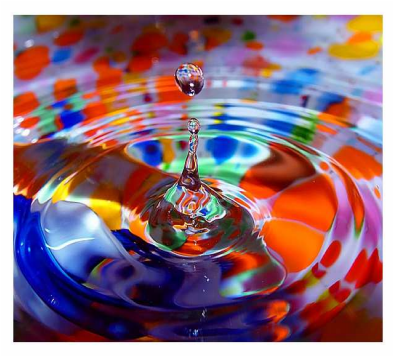

(a) Average score: 8.5714

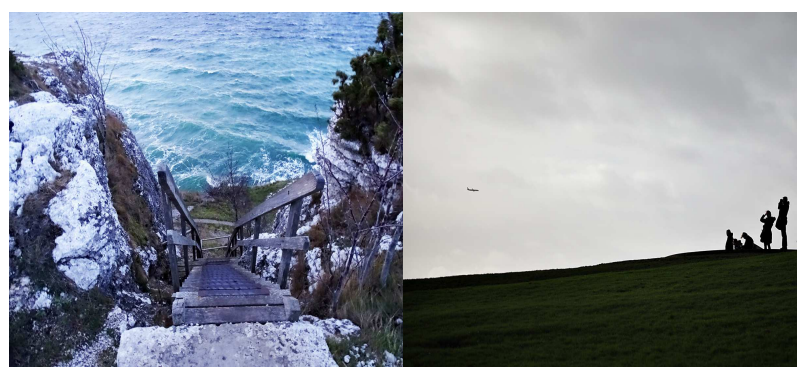

(c) Average score: 5.9804

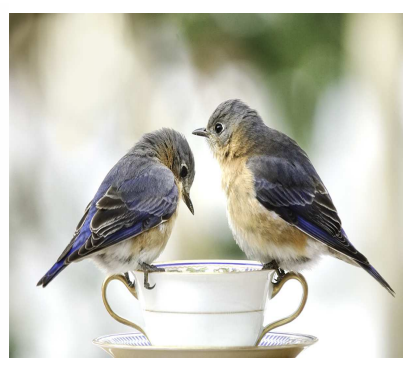

(b) Average score: 6.8824
Figure 1: Photos with ratings given by viewers collected from www.dpchallenge.com

In this work, we introduce a new approach of predicting image aesthetics using tags generated by the computer vision API of the Microsoft Azure Cognitive Services. The generated tags are mostly object names, but there are some context-related tags such as indoor, outdoor, etc. We use 250,000 images of the AVA dataset [21] where each of the images is associated with an aesthetic score on a 
scale ranging from $1-10$. We also use three types of aesthetic prediction models to understand the mapping between human understandable semantic features to the aesthetic score of every image. We show that our proposed idea of using tags for aesthetics prediction can produce comparative results close to the state-of-the-art methods and that is the main finding of this paper.

The rest of the paper is organized as follows. In Section 2 we discuss more related work on this topic. Section 3 provides the details of the used dataset and the proposed prediction models. In Section 4 we present the experimental results of image aesthetics prediction and conclude this paper in Section 5.

\section{RELATED WORKS}

In this section, we talk about the related works where the problem of image aesthetics analysis has been formulated as a classification or a regression problem. We review the hand-designed features and generic features proposed over the years to solve this problem. We also discuss the recent studies that show that convolutional neural network can be successfully applied for image aesthetics prediction achieving state-of-the-art results.

The approaches that formulate aesthetic quality assessment as a classification problem distinguish aesthetically pleasing and displeasing images. The hand-designed features that were proposed in the literature for appropriate representation of image aesthetic characteristics are as follows: Datta et al. [5] designed 56 visual features for each image based on intuition. Apart from considering visual cues like colorfulness, brightness, saturation, hue etc. they also considered features related to wavelet-based texture, size and aspect ratio, shape convexity, low depth of field etc and trained a statistical model to automatically classify images of having good or bad aesthetic quality. Ke et al. [13] used high-level semantic features to describe the spatial distribution of the high-frequency edges, color distribution, hue count, blur etc. for the classification task. Dhar et al. [9] proposed a different type of human-perceived high-level image attributes related to image configuration, the content of the image and the natural lighting conditions of the image, to predict image aesthetics and interestingness of the image. Nishiyama et al. [22] proposed an approach based on color harmony and bags of color patterns to deal with the complex color distribution of an image. They combined the color harmony feature along with blur, edges, and saliency features of the photos to improve their aesthetic classification performance. An alternative approach of using generic image descriptors such as GIST, Bag-ofVisual-Words descriptor, Fisher vector were proposed by Marchesotti et al. [19] which being able to implicitly encode the aesthetic characteristics of an image from SIFT information [16], could outperform traditional hand-designed features. Despite the success of the prior works using handcrafted features and generic features, in recent years several deep convolutional neural networks have been proposed for image aesthetics prediction. Lu et al. [17] proposed a double column $\mathrm{CNN}$ to improve aesthetic categorization using style attributes and semantic attributes. Two heterogeneous inputs, i.e., global and local views of an image, were fed to both the columns of the double column deep CNN, in order to capture both global and local characteristics of images.
On the other hand, the approaches that formulate aesthetic quality assessment as a regression problem focuses on finding the aesthetic scores using several data-driven machine learning techniques. Datta et al. [5] predicted the numerical aesthetics ratings by using Linear Regression (LR) on polynomial terms of the features. Bhattacharya et al. [1] used an interactive application, based on user-guided object segmentation and inpainting for extracting aesthetic features subsequently used for training a Support Vector Regression (SVR) model. Wu et al. [30] designed a new regression algorithm called support vector distribution regression (SVDR) and two separate learning strategies (RSL and LR) to tackle the difficulties in learning of a visual quality distribution prediction model. Leveraging the success of CNN architectures, Kao et al. [19] proposed a regression model based on $\mathrm{CNN}$ and showed impressive results. Later, Jin et al. [11] came up with a CNN based histogram prediction model that not only predicts the aesthetic score but also is able to obtain an aesthetically pleasing crop of an input image using the same regression model. Most recently, Murray et al. [20] showed that using only one deep CNN model (trained only for the distribution prediction task), three different kinds of tasks namely aesthetic quality classification, aesthetic score regression, and aesthetic score distribution prediction, can be solved.

However, none of the previous works focused what's inside the photos. In this work, we propose a novel approach of predicting image aesthetic score using machine-generated tags based on the content of the image. This research is the first trial that use object detection in aesthetics analysis tasks. Surprisingly, only machine generated tags can achieve comparable prediction performance to the state-of-the-art results.

\section{PROPOSED METHOD}

In this section, we explain the details of our procedure for mapping raw RGB images to aesthetics tags. We first describe the AVA dataset [21] and how we extracted the labels from the data. We also give details of the different models we used for determination of the aesthetic scores.

We denote each image by $X_{i}$ and the corresponding score by $s_{i}$. The score is usually in the range of 0 to 10 . We assume that the tags obtained from all the images in the dataset belong to a set $\mathcal{T}$. A generator of tag from images $g_{\text {tag }}: \mathbb{R}^{M \times N} \mapsto \mathcal{T}$. The predicted score for each image $X_{i}$ is given by $y_{i}$. The indicator function $\mathbb{1}(x)$ produces 1 if $x$ is true else 0 .

\subsection{Dataset}

We use the large-scale image database for Aesthetic Visual Analysis known as AVA dataset [21], which contains more than 255,000 images covering a wide variety of subjects on 1,447 challenges. These images along with a rich variety of meta-data were collected from an online community of photography amateurs such as www.dpchallenge.com [21]. We used all 255,494 images for our experimental purpose. For each image $X_{i}$, we have a distribution of user votes from score 1 to 10 . We compute the weighted average for these score distribution with votes as the weights to obtain a single real values score depicting the aesthetic rating of the image. 


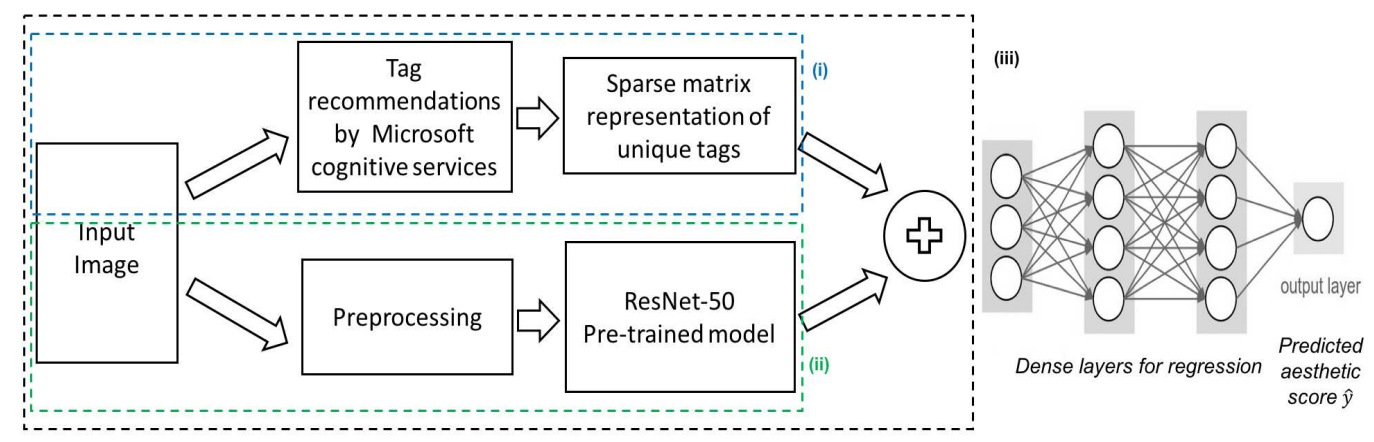

Figure 2: Proposed architecture

\subsection{Prediction model}

In this paper, our main focus is to illustrate the dependence of human understandable semantic features to the aesthetics of each image. More specifically, we try to find the relationship between machine generated tags and the aesthetic score of the image. We leverage Microsoft Azure Cognitive Services framework to generate tags of each image which we use for building regressor models for the aesthetic score. We mainly illustrate three kinds of models: (i) Naive Bayes model using only tag information, (ii) Convolutional Neural network(CNN) based score regression, and (iii) Combination of $\mathrm{CNN}$ and sparse tag information vector for score prediction. The details of the architectures are shown in Figure 2.

3.2.1 Naive Bayes using only tags. In this approach, we considered only the machine-generated tags to find its effect on the aesthetic scores. We opted for the naive Bayes regressor, where for each tag we find the average aesthetic scores of all the images having that tag. The mathematical formulation of the same is given as follows:

$$
p\left(y_{i} \mid t_{1}, t_{2}, \ldots, t_{n_{i}}\right)=p(y) \prod_{j=1}^{n_{i}} p\left(t_{j} \mid y_{i}\right),
$$

We assume an uninformative uniform prior on the aesthetic score distribution, and two sided exponential distribution,

$$
p\left(t_{j} \mid y_{i}\right)=\frac{\lambda}{2} \exp \left(-\lambda\left|y_{i}-\mu_{j}\right|\right)
$$

where $\mu_{j}=\frac{\sum_{i=1}^{N} s_{i} \mathbb{1}\left(t_{j} \in g_{\text {tag }}\left(X_{i}\right)\right.}{\sum_{i=1}^{N} \mathbb{1}\left(t_{j} \in g_{\text {tag }}\left(X_{i}\right)\right.}$ is the mean score for each tag across all images and $\lambda$ is the inverse mean of the distribution. With the above assumption of two sided exponential distribution, we get the mode of the aesthetic score distribution as,

$$
y^{*}=\frac{1}{n_{i}} \sum_{i=1}^{n_{i}} \mu_{j} .
$$

This gives a closed form solution to the aesthetic score prediction model from tag information and there is no iterative learning procedure involved in this method. While being very simple and straight-forward modeling, in the experimental section, we show that, it does a very good job of modeling the relationship between high-level human understandable tag information and aesthetic score, comparable to certain early methods in aesthetics prediction.

3.2.2 Convolutional Neural Network. It has been shown that residual networks [10] can produce state-of-the-art results in image classification tasks and the intermediate feature representations from the learned parameters of Residual Network (ResNet) can extract meaningful semantic knowledge about the content of the images. Thus these features have been used for various kinds of end-to-end image based tasks such as segmentation and depth estimation from a single image and other high-level computer vision tasks. We leverage these semantically meaningful features to extract aesthetic score from images. Although image aesthetics analysis is a highly subjective matter, our belief is that humans are aesthetically inclined to certain features in images whereas some other features decrease the attractiveness of an image. Given this, the aesthetic score prediction problem reduces to correlating features that improve or decrease aesthetic score of an image. To achieve the above mapping between semantically meaningful features and aesthetic score we deploy a multi-layer perceptron with single output depicting the real-valued score. Similar to recent works in deep models we leverage a large number of image data available from the AVA dataset [21] to learn generalizable features for the above mapping. This is obtained by simply minimizing the mean square error between the feed-forward prediction of the network and the ground-truth score while back-propagating the error gradients through the network and weight update using ADAM optimization method [14]. This method is similar to prior works but we use ResNet [10] features which are more expressive and can lead to better understanding of raw RGB to realvalued aesthetic scores. Although we have used only high-level fully connected features we believe there is room for improvement concatenating low-level, mid-level and high-level ResNet features similar to prior work; where we consider small details of the image like dots, lines, edges, gradients, pixel intensities or colours as low-level features; color histograms, texture or shape descriptors as mid-level features and features related to shapes and objects of the image as high level features. We resize each of the image in the AVA dataset [21] to $224 \times 224 \times 3$ so that it matches the Imagenet [8] dimensions and this stage is referred to as the pre-processing 


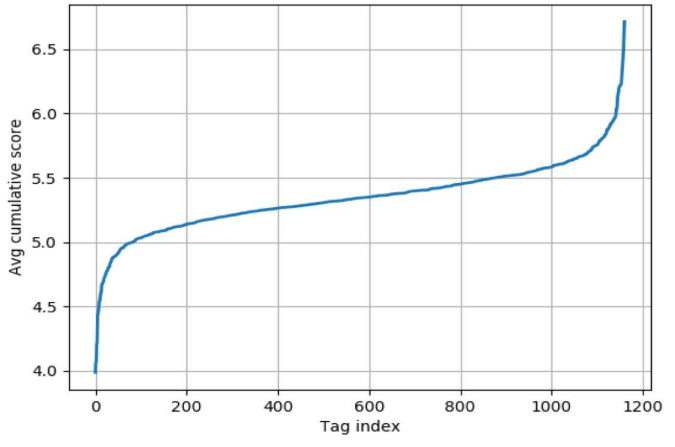

Figure 3: Relationship of tag index with average cumulative aesthetic scores

stage. Then, we use the ResNet-50 architecture pretrained on Imagenet dataset [8] and extract high-level features obtained from fully connected units.

3.2.3 CNN using tag spare vectors. In this approach, we concatenate both the features extracted by $\mathrm{CNN}$ and a sparse feature vector obtained from the tag generator usingMicrosoft Azure Cognitive Services. Although prior work has shown that combination of low level, mid-level, and high-level features results in the good performance of aesthetic score prediction, neither of these features were human understandable. In this method, we combine latent feature vectors from deep models and inspect how humanunderstandable features like object tags influence the aesthetics of an image. This is important because it gives a hint of interpretability as to why a certain image is aesthetically more appealing to the subjective human brain.

To achieve this we fuse features obtained from ResNet explained in the previous section in two ways: (i) early fusion, where we directly fuse the sparse feature vectors obtained from one-hot representation of tags in a particular image with real-valued ResNet features of size 2048, (ii) late fusion, where we first find an intermediate real-valued representation from the one-hot representation and then fuse it with the ResNet features.

\subsection{Visualizing dependence of tags on aesthetic score}

Figure 3 shows the relationship between the tag index with the average cumulative score, where we can see that in the middle region, the gradient is low and the randomness is greater. Therefore these tags affect the average cumulative score very little. However, in both of the sides, the gradient is quite high and the randomness is much lower, which means that the tags in those two regions affect the average aesthetic score in a great fashion.

\section{EXPERIMENTAL RESULTS}

In this section, we show the experimental results reported in the literature and the results obtained from the three architectures as explained above. Table 1 shows the mean square errors for each of the methods. The top four rows in Table 1 report the results
Table 1: Mean Square Error for different methods

\begin{tabular}{|c|c|}
\hline & MSE \\
\hline GIST linear-SVR & 0.5222 \\
\hline GIST rbf-SVR & 0.5307 \\
\hline BoVW SIFT linear-SVR & 0.5401 \\
\hline BoVW SIFT rbf-SVR & 0.5513 \\
\hline Kao et al. 2015 [12] & 0.4510 \\
\hline Jin et al. 2016 [11] & 0.337 \\
\hline Murray et al. 2017 [20] & 0.279 \\
\hline (i) Naive Bayes using only tags & 0.512 \\
\hline (ii) CNN based score regression & 0.3569 \\
\hline $\begin{array}{c}\text { (iii) Combination of CNN and } \\
\text { sparse tag information vector }\end{array}$ & $\mathbf{0 . 3 5 6 2}$ \\
\hline
\end{tabular}

obtained by combining the generic image descriptors, GIST [24], SIFT [16] and Bag-of-Visual-Words (BoVW) [4], along with the linear and non-linear Support Vector Regression (SVR) [28]. Note that these results are reported directly from the paper. Details of these methods can be found in [19], [12]. The last three rows show the result achieved by our proposed architectures. Overall it shows that our method of aesthetic score prediction using $\mathrm{CNN}$ and using the combination architecture performs almost comparable with the previous methods reported in the literature. Figure 4 shows the qualitative results and the correlation between the ground truth scores (GT) and the aesthetic scores predicted (pred) by our model (iii). We can see that the model can predict aesthetic scores close to the ground-truth scores. In Figure 5, however, we show the failure cases i.e. the images for which our proposed method failed to predict the aesthetic scores correctly. The ground truth scores (GT) and predicted scores (pred) are mentioned for each of the images.

\section{CONCLUSION}

In this paper, we proposed a novel idea of using tags for image aesthetics prediction. We formulated the problem as a regression problem with three kinds of models: (i) Naive Bayes model using only tag information, (ii) Convolutional Neural network (CNN) based aesthetic score regression, and (iii) A combinational architecture of $\mathrm{CNN}$ and sparse tag information vector for aesthetic score prediction. Experimental results on the AVA dataset, which is the benchmark dataset with the rich aesthetic rating, showed that our proposed idea performed comparably with the state-of-the-art results. Although it could not outperform the previously reported results on the same dataset, it is worth mentioning that, this is the very first attempt of using inferential information to predict the aesthetic score. We showed that even simple tag based naive regressor could produce quite good results for aesthetic score prediction. We believe that using a combination of low-level deep features combined with inferential information like tags, can be used to produce state-of-the-art systems that can be used to increase the number of views/likes on Social Networking Sites. 


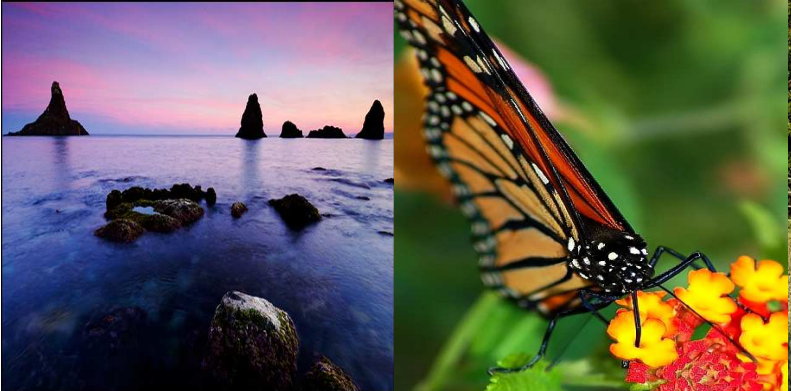

(a) GT: 6.530, Pred: 6.713

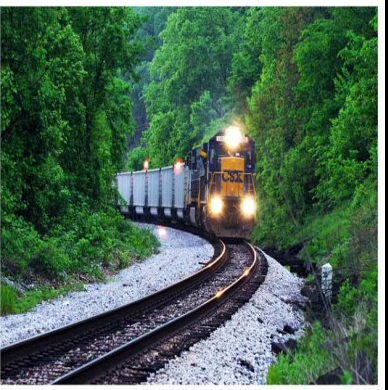

(e) GT: 5.522, Pred: 5.714 (b) GT: 6.370, Pred: 6.090

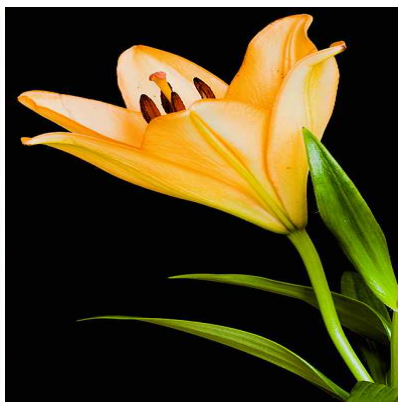

(f) GT: 6.191, Pred: 6.069

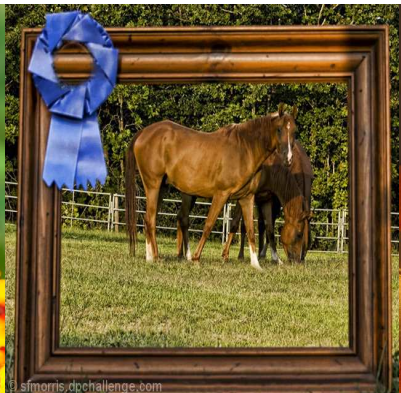

(c) GT: 5.383, Pred: 5.247

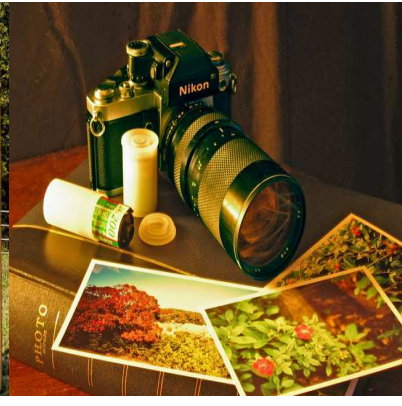

(d) GT: 6.530, Pred: 6.713

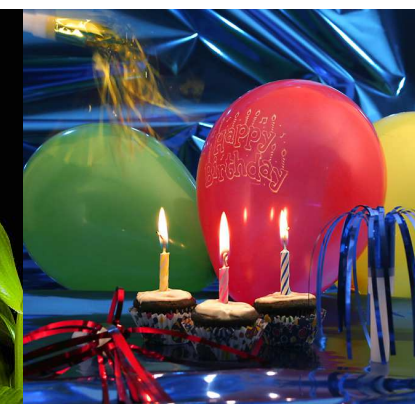

(g) GT: 5.207, Pred: 5.393

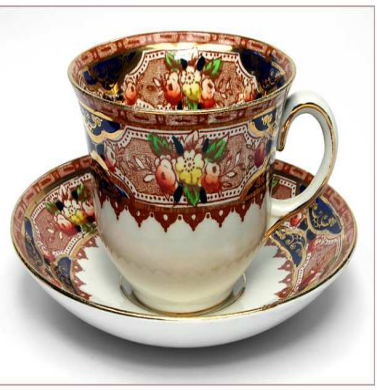

(h) GT: 5.841, Pred: 6.032

Figure 4: The ground truth scores (GT) and predicted scores (Pred) for some images in test set. The ground truth scores are shown in blue. The predicted scores are shown in green.

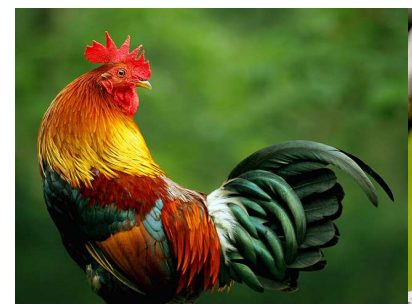

(a) GT: 7.596, Pred: 5.963

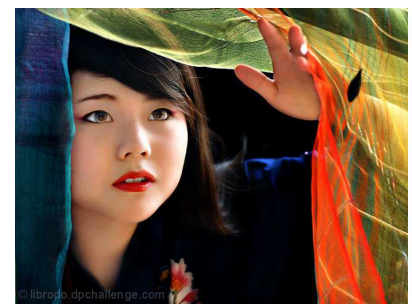

(c) GT: 7.857, Pred: 5.577

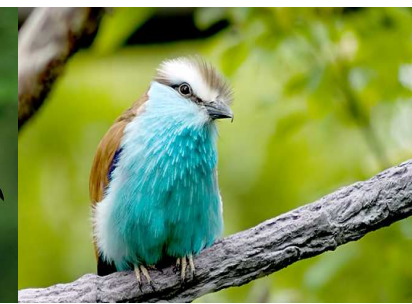

(b) GT: 6.459, Pred: 5.470

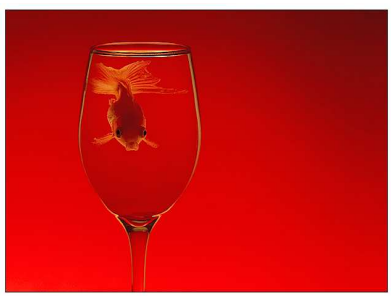

(d) GT: 7.321, Pred: 5.963

Figure 5: Failure cases of our aesthetic prediction model

\section{ACKNOWLEDGEMENTS}

This work was partially supported by the Grants-in-Aid for Scientific Research (no. 26700008) from JSPS, JST-CREST (JPMJCR1686), and Microsoft IJARC core13.

\section{REFERENCES}

[1] Subhabrata Bhattacharya, Rahul Sukthankar, and Mubarak Shah. 2010. A Framework for Photo-quality Assessment and Enhancement Based on Visual Aesthetics. In Proceedings of the 18th ACM International Conference on Multimedia (MM '10). ACM, New York, NY, USA, 271-280. https://doi.org/10.1145/1873951.1873990

[2] Simone Bianco, Luigi Celona, Paolo Napoletano, and Raimondo Schettini. 2016. Predicting Image Aesthetics with Deep Learning. In Advanced Concepts for Intelligent Vision Systems, Jacques Blanc-Talon, Cosimo Distante, Wilfried Philips, Dan Popescu, and Paul Scheunders (Eds.). Springer International Publishing, Cham, 117-125.

[3] Dan C. Ciresan, Ueli Meier, and Jürgen Schmidhuber. 2012. Multi-column Deep Neural Networks for Image Classification. CoRR abs/1202.2745 (2012). arXiv:1202.2745 http://arxiv.org/abs/1202.2745

[4] Gabriella Csurka, Christopher R. Dance, Lixin Fan, Jutta Willamowski, and CÃldric Bray. 2004. Visual categorization with bags of keypoints. In In Workshop on Statistical Learning in Computer Vision, ECCV. 1-22.

[5] Ritendra Datta, Dhiraj Joshi, Jia Li, and James Z. Wang. 2006. Studying Aesthetics in Photographic Images Using a Computational Approach. In Proceedings of the 9th European Conference on Computer Vision Volume Part III (ECCV'06). Springer-Verlag, Berlin, Heidelberg, 288-301. https://doi.org/10.1007/11744078_23

[6] Ritendra Datta, Jia Li, and James Z. Wang. 2007. Learning the Consensus on Visual Quality for Next-generation Image Management. In Proceedings of the 15th ACM International Conference on Multimedia (MM '07). ACM, New York, NY, USA, 533-536. https://doi.org/10.1145/1291233.1291364

[7] R. Datta, Jia Li, and J. Z. Wang. 2008. Algorithmic inferencing of aesthetics and emotion in natural images: An exposition, In Image Processing, 2008. ICIP 2008. 15th IEEE International Conference on. Image Processing, 2008. ICIP 2008. 15th IEEE International Conference on, 105-108. https://doi.org/10.1109/ICIP.2008.4711702

[8] J. Deng, W. Dong, R. Socher, L.-J. Li, K. Li, and L. Fei-Fei. 2009. ImageNet: A Large-Scale Hierarchical Image Database. In CVPR09.

[9] Sagnik Dhar, Vicente Ordonez, and Tamara L. Berg. 2011. High level describable attributes for predicting aesthetics and interestingness. 1657-1664. https://doi.org/10.1109/CVPR.2011.5995467 
[10] Kaiming He, Xiangyu Zhang, Shaoging Ren, and Jian Sun. 2015. Deep Residual Learning for Image Recognition. CoRR abs/1512.03385 (2015). arXiv:1512.03385 http://arxiv.org/abs/1512.03385

[11] B. Jin, M. V. O. Segovia, and S. SÃijsstrunk. 2016. Image aesthetic predictors based on weighted CNNs. In 2016 IEEE International Conference on Image Processing (ICIP). 2291-2295. https://doi.org/10.1109/ICIP.2016.7532767

[12] Yueying Kao, Chong Wang, and Kaiqi Huang. 2015. Visual aesthetic quality assessment with a regression model. In 2015 IEEE International Conference on Image Processing, ICIP 2015, Quebec City, QC, Canada, September 27-30, 2015. 1583-1587. https://doi.org/10.1109/ICIP.2015.7351067

[13] Yan Ke, Xiaoou Tang, and Feng Jing. 2006. The Design of High-Level Features for Photo Quality Assessment. In Proceedings of the 2006 IEEE Computer Society Conference on Computer Vision and Pattern Recognition - Vol ume 1 (CVPR '06). IEEE Computer Society, Washington, DC, USA, 419-426. https://doi.org/10.1109/CVPR.2006.303

[14] Diederik P. Kingma and Jimmy Ba. 2014. Adam: A Method for Stochastic Optimization. CoRR abs/1412.6980 (2014). arXiv:1412.6980 http://arxiv.org/abs/1412.6980

[15] Alex Krizhevsky, Ilya Sutskever, and Geoffrey E. Hinton. 2012. ImageNet Classification with Deep Convolutional Neural Networks. In Proceedings of the 25th International Conference on Neural Information Processing Systems - Volume 1 (NIPS'12). Curran Associates Inc., USA, 1097-1105. http://dl.acm.org/citation.cfm?id=2999134.2999257

[16] David G. Lowe. 2004. Distinctive Image Features from ScaleInvariant Keypoints. Int. 7. Comput. Vision 60, 2 (Nov. 2004), 91-110. https://doi.org/10.1023/B:VISI.0000029664.99615.94

[17] Xin Lu, Zhe L. Lin, Hailin Jin, Jianchao Yang, and James Z. Wang. 2015. Rating Image Aesthetics Using Deep Learning. IEEE Trans. Multimedia 17, 11 (2015), 2021-2034. https://doi.org/10.1109/TMM.2015.2477040

[18] Wei Luo, Xiaogang Wang, and Xiaoou Tang. 2011. Content-based photo quality assessment.. In ICCV, Dimitris N. Metaxas, Long Quan, Alberto Sanfeliu, and Luc J. Van Gool (Eds.). IEEE Computer Society, 2206-2213. http://dblp.uni-trier.de/db/conf/iccv/iccv2011.html\#LuoWT11

[19] Luca Marchesotti, Florent Perronnin, Diane Larlus, and Gabriela Csurka. 2011. Assessing the aesthetic quality of photographs using generic image descriptors.. In ICCV, Dimitris N. Metaxas, Long Quan, Alberto Sanfeliu, and Luc J. Van Gool (Eds.). IEEE Computer Society, 1784-1791. http://dblp.uni-trier.de/db/conf/iccv/iccv2011.html\#MarchesottiPLC11
[20] Naila Murray and Albert Gordo. 2017. A deep architecture for unified aesthetic prediction. CoRR abs/1708.04890 (2017). arXiv:1708.04890 http://arxiv.org/abs/1708.04890

[21] N. Murray, L. Marchesotti, and F. Perronnin. 2012. AVA: A large-scale database for aesthetic visual analysis. In 2012 IEEE Conference on Computer Vision and Pattern Recognition. 2408-2415. https://doi.org/10.1109/CVPR.2012.6247954

[22] M. Nishiyama, T. Okabe, I. Sato, and Y. Sato. 2011. Aesthetic quality classification of photographs based on color harmony. In CVPR 2011. 33-40. https://doi.org/10.1109/CVPR.2011.5995539

[23] P. Obrador, L. Schmidt-Hackenberg, and N. Oliver. 2010. The role of image composition in image aesthetics. In 2010 IEEE International Conference on Image Processing. 3185-3188. https://doi.org/10.1109/ICIP.2010.5654231

[24] Aude Oliva and Antonio Torralba. 2001. Modeling the Shape of the Scene: A Holistic Representation of the Spatial Envelope. Int. F. Comput. Vision 42, 3 (May 2001), 145-175, https://doi.org/10.1023/A:1011139631724

[25] Jose San Pedro, Tom Yeh, and Nuria Oliver. 2012. Leveraging User Comments for Aesthetic Aware Image Search Reranking. In Proceedings of the 21st International Conference on World Wide Web (WWW'12). ACM, New York, NY, USA, 439-448. https://doi.org/10.1145/2187836.2187896

[26] F. Simond, N. Arvanitopoulos, and S. SÃijsstrunk. 2015. Image aesthetics depends on context. In 2015 IEEE International Conference on Image Processing (ICIP). 3788-3792. https://doi.org/10.1109/ICIP.2015.7351513

[27] Karen Simonyan and Andrew Zisserman. 2014. Very Deep Convolutional Networks for Large-Scale Image Recognition. CoRR abs/1409.1556 (2014) arXiv:1409.1556 http://arxiv.org/abs/1409.1556

[28] Alex J. Smola and Bernhard Schölkopf. 2004. A Tutorial on Support Vector Regression. Statistics and Computing 14, 3 (Aug. 2004), 199-222. https://doi.org/10.1023/B:STCO.0000035301.49549.88

[29] Christian Szegedy, Wei Liu, Yangqing Jia, Pierre Sermanet, Scott E. Reed, Dragomir Anguelov, Dumitru Erhan, Vincent Vanhoucke, and Andrew Rabinovich. 2014. Going Deeper with Convolutions. CoRR abs/1409.4842 (2014) arXiv:1409.4842 http://arxiv.org/abs/1409.4842

[30] $\mathrm{Ou} \mathrm{Wu}$, Weiming $\mathrm{Hu}$, and Jun Gao. 2011. Learning to predict the perceived visual quality of photos. In 2011 International Conference on Computer Vision. 225-232. https://doi.org/10.1109/ICCV.2011.6126246

[31] Matthew D. Zeiler and Rob Fergus. 2013. Visualizing and Understanding Convolutional Networks. CoRR abs/1311.2901 (2013). arXiv:1311.2901 http://arxiv.org/abs/1311.2901 